\title{
In-situ Electrical Transport Measurements Combined with Scanning Transmission X-ray Microscopy
}

\author{
$\underline{\text { S. Finizio }}^{1, *}$, K. Zeissler ${ }^{2}$, G. Burnell ${ }^{2}$, C.H. Marrows ${ }^{2}$, and J. Raabe ${ }^{1}$ \\ 1. Swiss Light Source, Paul Scherrer Institut, 5232 Villigen PSI, Switzerland \\ 2. School of Physics and Astronomy, University of Leeds, Leeds LS2 9JT, United Kingdom \\ * Corresponding author, simone.finizio@psi.ch
}

The interpretation of electrical transport measurements on novel sample systems such as e.g. magnetic skyrmions in multilayer systems [1-4] usually requires the combination of these measurements with imaging techniques, to allow for the correlation of the magnetic state with the recorded transport measurement (see e.g. Refs. [3,4]).

Scanning transmission $\mathrm{x}$-ray microscopy (STXM) is a transmission microscopy technique that employs a Fresnel zone plate to focus a monochromatic $\mathrm{x}$-ray beam onto a spot on a sample, recording the $\mathrm{x}$-ray intensity transmitted across the sample with a suitable detector (e.g. an avalanche photodiode, or a photomultiplier tube). A STXM image is formed by scanning the sample with a piezoelectric stage (usually combined with a laser interferometer stage to improve the positioning precision), and recording the transmitted $\mathrm{x}$-ray intensity at each point of the scan. This technique can be combined with the x-ray magnetic circular dichroism (XMCD) effect to obtain images of the magnetic configuration of a magnetic sample.

Here, we describe a setup, currently installed at the PolLux (X07DA) endstation of the Swiss Light Source, for the combined XMCD-STXM imaging and in-situ electrical transport measurement of magnetic materials.

The setup reported here, shown schematically in Fig. 1, employs a magnetic sample fabricated on an xray transparent substrate with patterned electrical contacts specific for the required transport measurement. Here, we will describe, as an example, the case of a Hall voltage measurement.

The transport measurement setup shown schematically in Fig. 1 employs a Keithley 6221 current source for the generation of an AC electrical current, which is injected across the magnetic sample. The Hall voltage generated by the injection of such current is then measured using a Zürich Instruments HF2LI lock-in amplifier, locked to the frequency of the AC current generated by the current source, in the differential mode. This setup can be easily integrated with the magnet installed at the PolLux endstation, allowing for the combined STXM and transport measurement of the sample under either in-plane or outof-plane magnetic fields. Furthermore, this setup was integrated with the control system of the Swiss Light Source and with the control software of the PolLux endstation, enabling the full automation of both the imaging and the transport measurements.

As a proof-of-principle measurement, a nanostructured disc of a $\mathrm{Pt} / \mathrm{Co} / \mathrm{Ir}$ stack stabilizing a perpendicularly magnetized state (for details on the sample preparation see Ref. [3]) was lithographically patterned on top of a $\mathrm{Si}_{3} \mathrm{~N}_{4}$ membrane and electrically contacted in a Hall cross geometry. This disc was then measured with the setup described above. Different out-of-plane magnetic fields were applied to the sample and, for each field step, an XMCD-STXM image, together with a reading of the Hall voltage, was recorded. For each field step, around 100 samples of the Hall voltage were recorded at a time interval of $10 \mathrm{~ms}$ between each consecutive point. The current generator was set at an operating frequency of $17 \mathrm{~Hz}$. The result of this measurement is shown in Fig. 2, where the switching is clearly observable both in the transport measurements, and in the XMCD-STXM images.

The proof-of-principle measurements reported here demonstrate that the combination of a lock-in based electrical transport measurement setup with a STXM instrument is feasible, and allows for the quasi- 
simultaneous acquisition of magnetic images and of electrical transport measurements of a magnetic sample, therefore providing a powerful input to the interpretation of transport measurements in novel sample systems. Furthermore, XMCD-STXM imaging has an additional advantage compared to competing techniques, as this technique provides direct images of the magnetic configuration of the material instead of indirect images of the magnetic configuration (e.g. magnetic force microscopy provides images based on the stray field generated by the sample, increasing the difficulties in determining the size of the magnetic domains [4]).

In conclusion, we report here on an experimental setup that enables the in-situ measurement of both the magnetic configuration of a nanostructured sample, and the measurement of its electrical transport properties such as e.g. the Hall voltage. This setup provides a powerful input for the interpretation of transport measurements in magnetic sample systems, as it allows one to directly correlate the electrical measurements with a direct image of the magnetic state of the sample. The capabilities of the instrumentation reported here were demonstrated by a proof-of-principle measurement of the switching of a Pt/Co/Ir nanostructured disc, and by the results reported in Ref. [3], where the transport properties of a magnetic skyrmion stabilized in a Pt/Co/Ir nanostructured disc were measured [5].

\section{References:}

[1] A. Fert et al., Nature Nanotechnology 8, 152 (2013)

[2] N. Nagaosa and Y. Tokura, Nature Nanotechnology 8, 899 (2013)

[3] K. Zeissler et al., arXiv:1706.06024 (2017)

[4] D. Maccariello et al., Nature Nanotechnology, doi:10.1038/s41565-017-0044-4 (2018)

[5] The authors acknowledge funding from the EU Horizon 2020 MAGicSky project (Grant No. 665095)

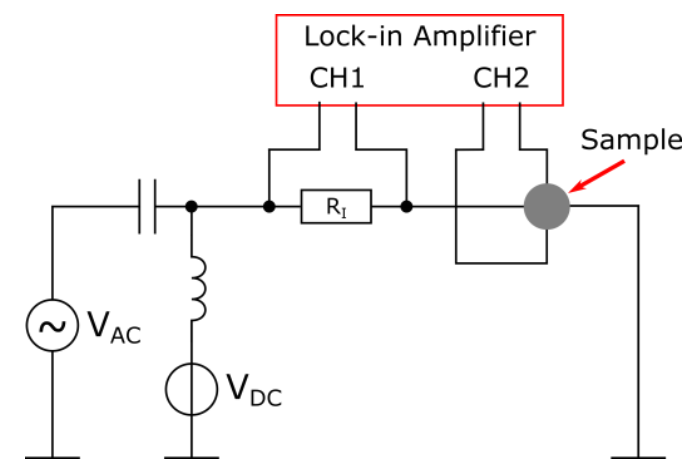

Figure. 1. Schematic overview of the transport measurement setup. The first channel of the lock-in amplifier is employed to measure the current injected across the sample (via a reference resistor), while the second channel is employed to measure the Hall voltage generated across the sample.

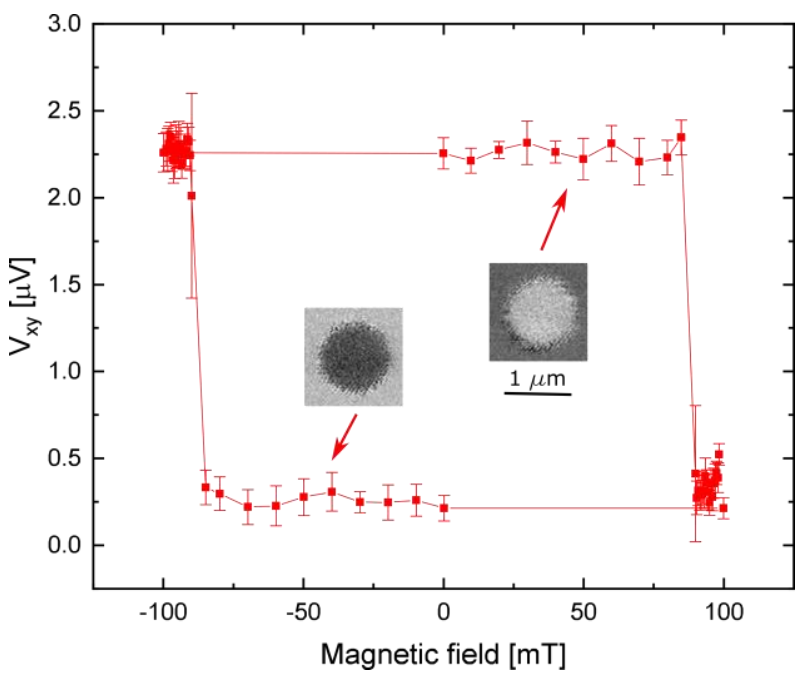

Figure. 2. Combined STXM and transport measurement of a nanostructured $\mathrm{Pt} / \mathrm{Co} / \mathrm{Ir}$ disc as a function of an applied external magnetic field. A clear switching of the magnetic state with the field can be observed both in the transport data and in the corresponding XMCD-STXM images. 